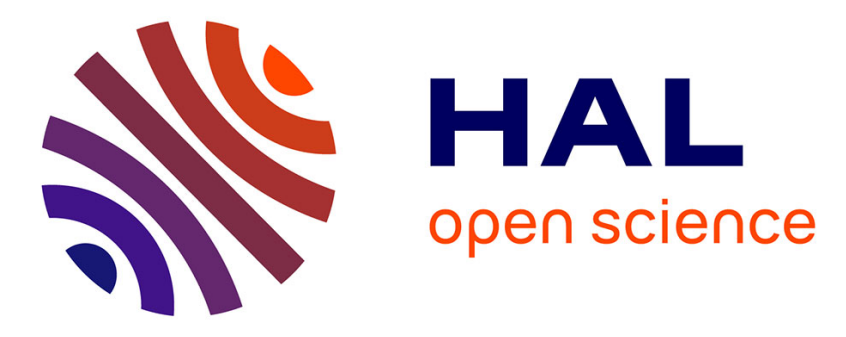

\title{
Transient gratings on a-GaAs under laser annealing conditions
}

\author{
W. Marine, J. Marfaing, F. Salvan
}

\section{To cite this version:}

W. Marine, J. Marfaing, F. Salvan. Transient gratings on a-GaAs under laser annealing conditions. Journal de Physique Lettres, 1983, 44 (7), pp.271-278. 10.1051/jphyslet:01983004407027100 jpa00232192

\section{HAL Id: jpa-00232192 https://hal.science/jpa-00232192}

Submitted on 1 Jan 1983

HAL is a multi-disciplinary open access archive for the deposit and dissemination of scientific research documents, whether they are published or not. The documents may come from teaching and research institutions in France or abroad, or from public or private research centers.
L'archive ouverte pluridisciplinaire HAL, est destinée au dépôt et à la diffusion de documents scientifiques de niveau recherche, publiés ou non, émanant des établissements d'enseignement et de recherche français ou étrangers, des laboratoires publics ou privés. 
Classification

Physics Abstracts

$42.60-61.40-61.50 \mathrm{C}$

\title{
Transient gratings on a-GaAs under laser annealing conditions
}

\author{
W. Marine, J. Marfaing and F. Salvan \\ Faculté des Sciences de Luminy, Département de Physique (*), Case 901, \\ 13288 Marseille Cedex 9, France
}

(Reçu le 24 novembre 1982, révisé le 10 février 1983, accepté le 14 février 1983)

\begin{abstract}
Résumé. - La diffraction de réseaux transitoires permet d'étudier la dynamique des processus de cristallisation de a-GaAs dans le domaine des nanosecondes. En faisant varier la densité de puissance d'irradiation $W$, on observe deux régimes :

$-3,5 \mathrm{MW} / \mathrm{cm}^{2}<W<7,5 \mathrm{MW} / \mathrm{cm}^{2}$ : cristallisation sans passage par une phase liquide;

$-7,5 \mathrm{MW} / \mathrm{cm}^{2}<W<15 \mathrm{MW} / \mathrm{cm}^{2}$ : passage par zone fondue avant la cristallisation.

Le processus de diffusion des porteurs libres joue un rôle important dans les mécanismes de recuit laser.
\end{abstract}

\begin{abstract}
Transient gratings are used to study the dynamical processes involved in a-GaAs crystallization on the nanosecond scale. When the irradiation power density is varied, two regimes are observed :

- $3.5 \mathrm{MW} / \mathrm{cm}^{2}<W<7.5 \mathrm{MW} / \mathrm{cm}^{2}$ : crystallization occurs without melting;

$-7.5 \mathrm{MW} / \mathrm{cm}^{2}<W<15 \mathrm{MW} / \mathrm{cm}^{2}$ : one observes a melted zone before crystallization.
\end{abstract}

Free carrier diffusion is a very efficient mechanism in these laser annealing conditions.

\section{Introduction.}

Transient gratings produced by the interference of two coherent light beams have been used previously to study carrier diffusion and bulk or surface recombination processes [1-3]. These studies have been reported for low excitation conditions or near melting threshold and damage conditions as well $[4,5]$. They are of fundamental and technological interest in the area of pulsed laser annealing of semiconductors. In this letter we report results on both self-diffraction and $\mathrm{cw}$ laser probe diffraction measurements on transient gratings produced in a-GaAs films under high excitation power. We will show that this technique gives a better insight on the mechanisms involved in laser crystallization of amorphous semiconductors.

\section{Experimental results.}

In our experiments we used a-GaAs films $0.8 \mu$ thick deposited by RF sputtering onto a glass substrate. The values of the absorption coefficient $\left(\alpha=8 \times 10^{4} \mathrm{~cm}^{-1} ; 4 \times 10^{4} \mathrm{~cm}^{-1}\right)$ and of the refractive index $(n=4.6 ; 4.1)$ for a- and c-GaAs respectively were determined by

(*) ERA CNRS 373. 
ellipsometric measurements. For our experimental arrangement we used a $\mathrm{Q}$ switched ruby laser $(h v=1.78 \mathrm{eV})$ with a pulse duration $\tau_{\mathrm{L}}=40 \mathrm{~ns}(\mathrm{FWHM})$. The light beams are separated by a beam splitter into two equal intensity beams with the same polarization which combined at a small angle, so that the grating period $(\Lambda=30 \mu)$ was large in comparison with carrier and thermal diffusion length (about $1 \mu \mathrm{s}$ ). We used neutral density filters in order to get the energy density range $0.1<E<1 \mathrm{~J} / \mathrm{cm}^{2}$. This was measured by a Joulemeter and controlled by calibrated PIN diode $\mathrm{PD}_{1}$. The time resolved intensity of the first order diffracted beam was observed with another PIN diode $\mathrm{PD}_{2}$ or a photomultiplier. The signal was displayed on a fast memory oscilloscope. A delay line allowed the observation of the shape of the excitation pulse provided by $\mathrm{PD}_{1}$ on the same trace. The excitation pulse was focused on a $1.5 \mathrm{~mm}$ diameter spot (FWHM). We observed either the self-diffraction during the laser pulse or the diffraction of a cw He-Ne laser probe focused on a $300 \mu$ diameter spot. The irradiated zones were examined finally either with optical microscopy (OM) or scanning electron microscopy (SEM).

The minimum power density at which crystallization occurs was observed at $W=3.5 \mathrm{MW} / \mathrm{cm}^{2}$. Above this threshold crystallites 1 to $3 \mu$ large according to the excitation energy were examinated with OM or SEM. The width of crystallized zone extended about $10 \mu$. Laser damage in the zone centre was observed at $W=15 \mathrm{MW} / \mathrm{cm}^{2}$. For $W>15 \mathrm{MW} / \mathrm{cm}^{2}$ vaporization of GaAs occurred.

Figure $1 a$ shows the typical oscilloscope traces of both first order self-diffracted signal $S$ and excitation pulse $L$ provided by $\mathrm{PD}_{1}$. One can note that for $W<7.5 \mathrm{MW} / \mathrm{cm}^{2}, S$ reproduces the

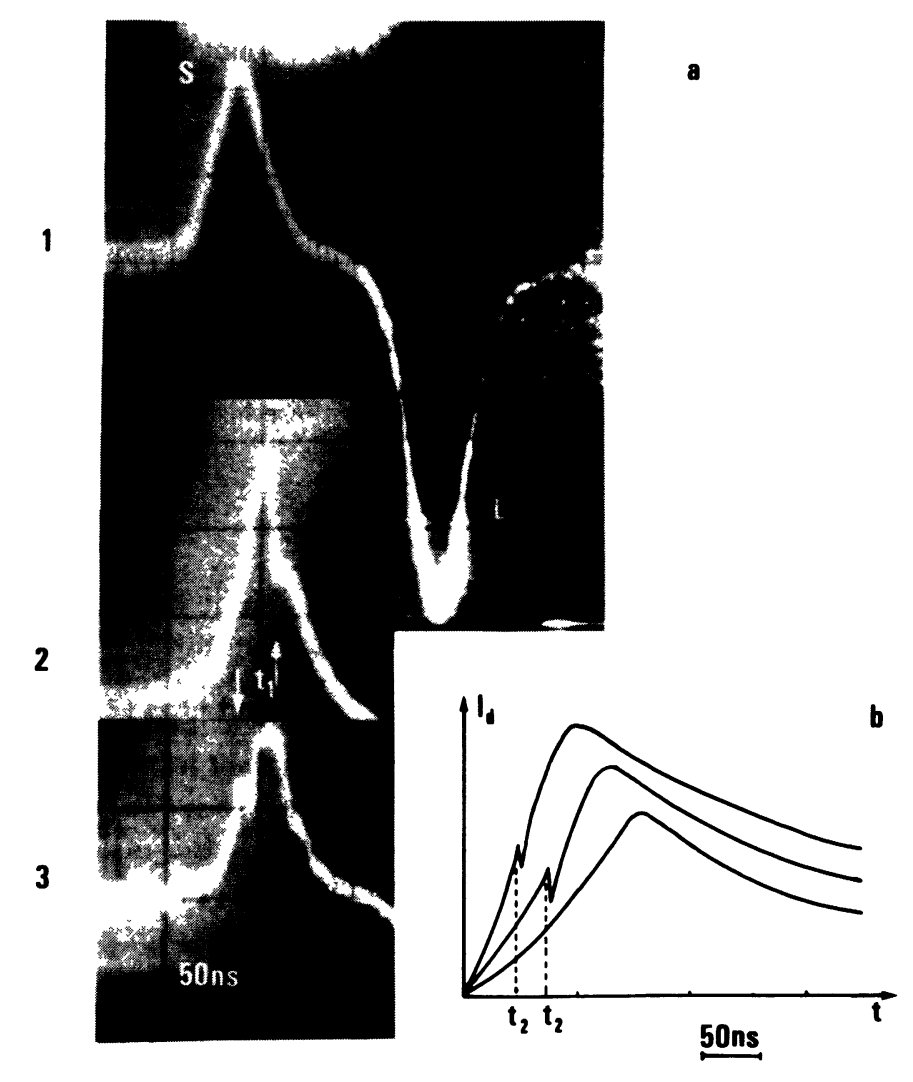

Fig. 1. - a) Typical oscilloscope traces of the first order self-diffracted signal $S$ for different excitation powers $W<7.5 \mathrm{MW} / \mathrm{cm}^{2}(1) ; 8.75 \mathrm{MW} / \mathrm{cm}^{2}(2) ; 12.5 \mathrm{MW} / \mathrm{cm}^{2}(3) . L$ is the laser pulse signal. $b$ ) Evolution of the diffracted $\mathrm{He}-\mathrm{Ne}$ light under the same excitation powers. 
shape of the excitation signal without any discontinuity (SP-CR). In the power range

$$
7.5 \mathrm{MW} / \mathrm{cm}^{2}<W<15 \mathrm{MW} / \mathrm{cm}^{2} \text { (LP-CR), }
$$

one observes, besides a maximum, a dip caused by a discontinuity in the $S$ signal. This dip occurs at shorter times $t_{1}$ in the pulse when one increases $W$.

When the grating diffraction was checked via the He-Ne probe, the diffracted light presented the time evolution behaviour indicated in figure $1 b$. For $W<7.5 \mathrm{MW} / \mathrm{cm}^{2}$, no dip was observed. For $7.5 \mathrm{MW} / \mathrm{cm}^{2}<W<15 \mathrm{MW} / \mathrm{cm}^{2}$, a discontinuity occurred at time $t_{2}$ and under the same excitation conditions, within the limits of our resolution $t_{2}=t_{1}$. We observed diffracted intensity at longer times after the excitation pulse and in the whole range where crystallization was present ; the diffracted signal stabilized at a constant value at times of the order of a few $100 \mathrm{~ns}$. This long time signal corresponds to the periodically amorphous-crystallized zones created by the irradiation. One can also note that the broad maximum signal intensity shifted towards shorter times when $W$ was increased.

\section{Discussion.}

The spatially modulated generation rate of free carriers is written as

$$
G(x, z, t)=(1-R) \frac{\alpha I_{0}}{h v} F(t) \mathrm{e}^{-\alpha z}\left(1+\cos \frac{2 \pi x}{\Lambda}\right),
$$

where $R$ is the reflection coefficient, $I_{0}$ and $F(t)$ are the radiation intensity and the time dependence of the excitation pulse, $x$ and $z$ are the coordinates respectively along the surface and in laser propagation direction. The periodic modulation of the dielectric constant occurs via various mechanisms namely non equilibrium free carrier concentration, lattice temperature $T_{1}$ changes, free carrier absorption, etc... Each mechanism induces changes $\Delta n$ and $\Delta k$ in $n$ and $k$. The diffracted intensity in first order is then written as [6]

$$
I_{\mathrm{d}}=I_{0} F(t) \cdot T J_{1}^{2}(\Phi+i a)
$$

where $T$ is the transmission function and $J_{1}$ is the first order Bessel function of a complex argument. $2 \Phi=2 \pi \Delta n d / \lambda$ and $2 a=2 \pi \Delta k d / \lambda$ are the full modulation depth of respectively the phase and amplitude, $d$ is the active thickness of the sample and $\lambda$ is the laser wavelength.

In order to explicit the different contributions $\Delta n, \Delta k$, we need to first describe the evolution of $T_{1}$ and of the free carrier density $N$ which are dependent on the time $t$ and the depth $z$. We thus look at the coupled equations :

$$
\begin{aligned}
\frac{\partial N}{\partial t} & =G\left(N, T_{1}, z, t\right)-\frac{N}{\tau\left(N, T_{1}\right)}+\frac{\partial}{\partial z}\left[D_{\mathrm{a}}\left(N, T_{1}\right) \frac{\partial N}{\partial z}\right] \\
\rho C\left(T_{1}\right) \frac{\partial T_{1}}{\partial t} & =G^{*}\left(N, T_{1}, \cdot z, t\right)+\frac{\partial}{\partial z}\left[K\left(N, T_{1}\right) \frac{\partial T_{1}}{\partial z}\right]
\end{aligned}
$$

$\tau$ is the free carrier lifetime. $D_{\mathrm{a}}$ is the ambipolar diffusion coefficient and $K$ the thermal conductivity. $G^{*}$ describes the heat generation rate while $\rho$ and $C$ are respectively the mass density and the specific heat.

In our experiments we observed the superposition of two gratings, a free carrier and a thermal one. In the most general case, the grating is mixed (amplitude and phase) $[1,4,6,7]$. At the beginning of excitation pulse $t<t_{1}$, when we can neglect the change of lattice temperature the grating is due to free carriers. We can show that this grating can be considered as a phase one because 
$\Phi / a=\Delta n / \Delta k \sim \omega \tau \gg 1$ where $\omega \sim 2.7 \times 10^{-15} \mathrm{~s}^{-1}$ is circular frequency of the probe light and $\tau \sim 10^{-14} \mathrm{~s}^{-1}$ is relaxation time.

When $T_{1}$ increases as recombination takes place, the periodic distribution of $T_{1}$ is then governed by equation (4), and the predominant factor of changes in $\Delta n$ and $\Delta k$, with constant power of excitation, is the change of lattice temperature, which corresponds to transient thermal grating.

In time $t \sim t_{1}$, before the dip, the ratio $\Phi / a$ is about 5 . This was evaluated from separate measurements on transmission of the sample and estimated $\Delta n$ for temperature near the melting point (1 $500 \mathrm{~K}$ ) from Cardona's results for $\mathrm{c}-\mathrm{GaAs}: \mathrm{d} n / \mathrm{d} T_{1}=5.4 \times 10^{-5} n^{\circ} \mathrm{C}^{-1}$ [8]. This $\Phi / a$ value is large enough to allow us to neglect the influence of the spatial transmission modulation and we thus can consider our grating before the dip as a thermal phase grating. Another point should be mentioned concerning the analysis of $I_{\mathrm{d}}$ in equation (2). A grating might be considered as a thin grating (Raman-Nath region) as long as the following condition is observed [9]

$$
Q=\frac{2 \pi \lambda d}{n \Lambda^{2}}<0.5
$$

and as long as $\Phi$ is not too large. If $\Phi$ is very large, then the Raman-Nath criterion takes the following form $Q \Phi<1$.

In our experimental conditions we have $Q \sim 8.4 \times 10^{-4} \ll 0.5$ and $Q \Phi<1$. At high carrier densities, e-h lifetime is governed by Auger recombination $\tau=1 / \gamma N^{2}$ with $\gamma=10^{-31} \mathrm{~cm}^{6} \mathrm{~s}^{-1}$ [10-11]. In this case we obtain the maximum free carrier concentration $N$ from the simple equation $\mathrm{d} N / \mathrm{d} t=G-\gamma N^{3}=0$. The generation rate $G$ is given by $(1-R) W / h v z$, where $z$ is the depth where energy is released. If the e-h diffusion length $L_{\mathrm{D}}$ is very large, then $z=d$, where $d$ is thickness of the sample. If $L_{\mathrm{D}}$ is negligible, then $z=\alpha^{-1}$. A calculation of $N$ values in the two limiting cases gives a maximum of $N=1.6-5 \times 10^{20} \mathrm{~cm}^{-3}$. The different mechanisms for the change of $\Delta n$ in $\mathrm{GaAs}$ under high density of free carriers have been estimated in the literature [12-14]. The maximum value of $\Delta n$ is about 0.1 with typical concentration $N \sim 10^{20} \mathrm{~cm}^{-3}$. Hence the maximum $\Phi$ value is $\sim 0.7$. It is smaller than $\Phi=1.9$ which corresponds to the first maximum of the Bessel function. In this case the existence of a sharp discontinuity in the diffracted signal cannot be interpreted in terms of a variation of the Bessel function argument associated with free carrier grating. We recall that $S$ reveals an abrupt change in the refractive index and further in time a strong increase of the diffracted light (Fig. 1).

In the case of a thermal grating, the parameter $\Phi$ depends on the temperature in their following way :

$$
\Phi=\frac{\pi}{\lambda} \int_{0}^{d} \frac{\mathrm{d} n}{\mathrm{~d} T_{1}}\left[T_{1}(0, z, t)-T_{1}\left(\frac{\Lambda}{2}, z, t\right)\right] \mathrm{d} z
$$

The temperature evolution during and after the excitation pulse can be described using the diffusion equation (4). The numerical resolution of this equation can be performed in the framework of the model used by Baeri et al. [15]. Before melting the typical change in $T_{1}$ versus time is as follows : the temperature exponentially increases, passes through a maximum and monotonically decreases.

To our knowledge, the variations of the refractive index versus the temperature above a few $100^{\circ} \mathrm{C}$ have not been experimentally determined for a-GaAs. If we suppose that the variations of the refractive index are analogous to those of $\mathrm{Si}$ [16], the variation of the Bessel function argument as a function of time has also to be monotonous and that cannot explain the sharp dip observed at $t_{1}$, during the excitation (Fig. 1).

As will be discussed now the dip can only be explained by a solid-liquid phase transition that occurs at the time $t_{1}$. Indeed, the solid-liquid phase transition of semiconductors is a first order phase transition [17], so that after melting we observe a rapid change in the optical constants, 
especially in the refractive index. This appears on the curve $n=f(T)$ as a discontinuity in $n$ values, which is represented schematically in figure 2 . This hypothesis is compatible with the existence of the dip which can thus be related to a solid-liquid transformation.

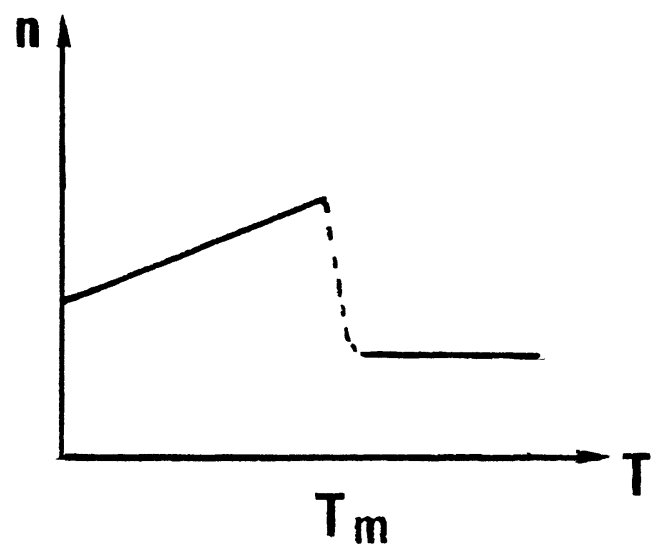

Fig. 2. - Schematic evolution of the refractive index $n$ versus temperature.

It is worth noticing that similar changes observed in reflectivity measurements have been reported by some authors [18-20]. One can observe the same anomalies, i.e. a dip in the high reflectivity phase.

In order to go further in our interpretation, we calculated the absorbed energy $E_{\mathrm{th}}$ in the GaAs sample between the beginning of laser pulse and the time $t_{1} . E_{\mathrm{th}}$, which we define as the melting threshold, is equal to

$$
(1-R) I_{0} \int_{0}^{t_{1}} F(t) \mathrm{d} t
$$

We plotted $E_{\text {th }}$ versus the excitation power density $W$ (Fig. 3) and used the results to calculate the width of the zone where $T_{1}$ is near the melting point $T_{\mathrm{m}}$.

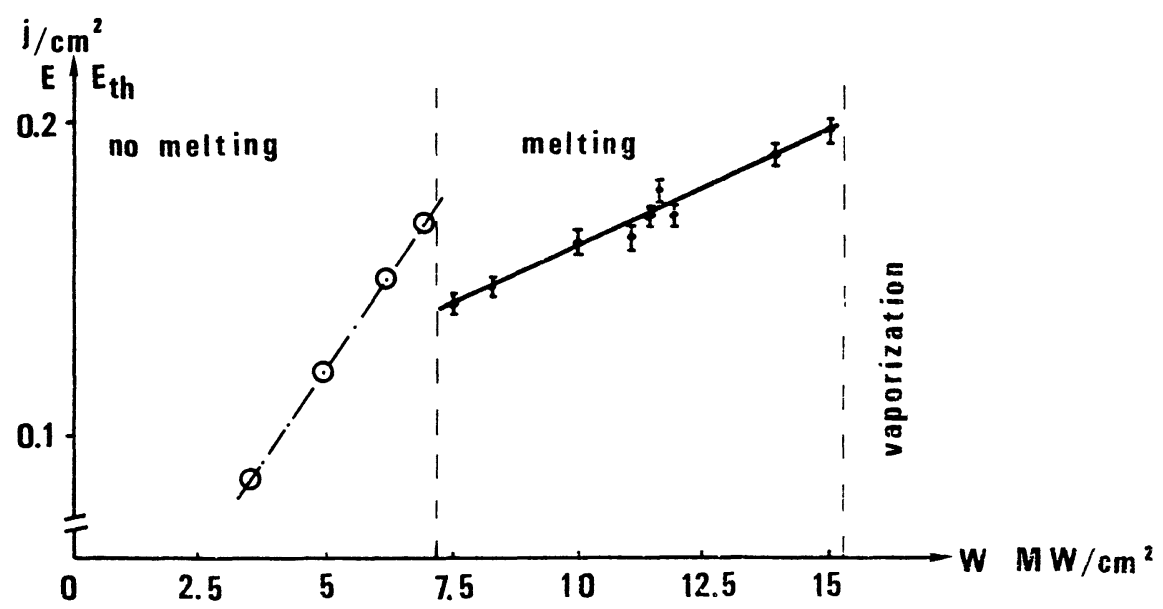

Fig. 3. - Calculated melting threshold $E_{\mathrm{th}}(\Phi)$ versus power excitations. The points $(\odot)$ correspond to the absorbed energy $E$ in the sample in the range $W<7.5 \mathrm{MW} / \mathrm{cm}^{2}$. 
3.1 For $7.5 \mathrm{MW} / \mathrm{cm}^{2}<W<15 \mathrm{MW} / \mathrm{cm}^{2}$. - We observed the melting threshold. Under such an excitation rate, the free carrier concentration reaches the extreme value $\sim 5 \times 10^{20} \mathrm{~cm}^{-3}$, and e-h lifetime is $\tau \approx 100 \mathrm{ps}$. When one deals with so high $N$ concentration carriers, $N>10^{19} \mathrm{~cm}^{-3}$, the ambipolar diffusion coefficient $D_{\mathrm{a}}$ could be strongly dependent of $N$ because the carriers are degenerate. Young et al. have treated this problem while taking into account the $N, T_{\mathrm{e}}, T_{1}$ dependences of $D_{\mathrm{a}} \cdot T_{\mathrm{e}}$ is the electronic temperature which could be different from $T_{1}$ at such high carrier densities [21].

In our experimental conditions, the diffusion length $L_{\mathrm{D}}=\left(D_{\mathrm{a}} \tau\right)^{1 / 2}$ is much larger than the absorption characteristic length $\alpha^{-1}$. In this case, within a certain error (about $15 \%$ ), it is considered that after free carrier diffusion, the energy which has been absorbed before melting, is homogeneously redistributed into the total thickness $L=L_{\mathrm{D}}+L_{\mathrm{T}}$ where $L_{\mathrm{T}}$ is the thermal diffusion length. We can estimate the minimal zone thickness where the temperature is near melting point $T_{\mathrm{m}}$ using the simple equation :

$$
L=\frac{E_{\mathrm{th}}}{\rho C(T)\left(T_{\mathrm{m}}-300\right)} .
$$

One should remark that this estimation neglects the heat of fusion. However as soon as melting occurs even on a very thin melted zone, an abrupt change in $\Delta n$ would provoke a discontinuity in the diffracted signal.

Under high density excitation $\left(W \sim 15 \mathrm{MW} / \mathrm{cm}^{2}\right)$, we observe in the diffracted pulse the dip $\sim 5 \mathrm{~ns}$ after the beginning of the excitation pulse. In these conditions, $L$ evaluated from (6) gives $L \simeq 0.68 \mu$, while $L_{\mathrm{T}} \simeq 0.22 \pm 0.03 \mu$ can be estimated from $L_{\mathrm{T}} \simeq(2 K(T)) t / \rho C(T)^{1 / 2}$ and equation (4). This gives us $L_{\mathrm{D}}=L-L_{\mathrm{T}} \simeq\left(D_{\mathrm{a}} \tau\right)^{1 / 2} \simeq 0.46$ and $D_{\mathrm{a}} \sim 52 \pm 8 \mathrm{~cm}^{2} \mathrm{~s}^{-1}$ for a carrier concentration of $5 \times 10^{20} \mathrm{~cm}^{-3}$ and temperature near $T_{\mathrm{m}}$. In the calculation we have used the following GaAs parameters : $\rho=5.31 \mathrm{~g} \mathrm{~cm}^{-3} ; T_{\mathrm{m}_{1}}=1511 \mathrm{~K} ; R=0.4$ [22]; $K(T) \simeq 0.425(T / 300)^{-1.1} \mathrm{~W} / \mathrm{cm} \mathrm{K}[23] ; C(T)=0.335+8.09 \times 10^{-2}(T-400) \mathrm{J} / \mathrm{gK}$ for $T>400 \mathrm{~K}[24]$.

This estimated $D_{\mathrm{a}}(N)$ value may be compared with $D_{0}=20 \mathrm{~cm}^{2} \mathrm{~s}^{-1}$ [25] for non degenerate carriers in c-GaAs. We can see that this value $D_{\mathrm{a}}(N)$ is much larger than $D_{0}$. This is due to the carrier concentration and temperature dependences of $D_{\mathrm{a}}(N, T)$, and it is in reasonable agreement with the conclusion of Young et al. [21]. To our knowledge, it does not exist in the literature on GaAs an experimental value of $D_{\mathrm{a}}$ for high temperature and high excitation conditions. However similar results have been reported by Auston and Shank [26] in the case of degenerate carriers in $\mathrm{Ge}$.

At this stage, we would make the following comments. Our analysis is based on the concentration dependence of $D_{\mathrm{a}}$ under high excitation conditions, but it is important to emphasize that the plasma expansion [27] might be important. This expansion is obtained from the internal pressure of the dense e-h plasma. This pressure is strongly dependent on the carrier concentration and the critical density $N^{*}, P \sim\left(N / N^{*}\right)^{2 / 3} N$ [30] $\left(N^{*}\right.$ of $\left.\mathrm{GaAs} \sim 10^{16} \mathrm{~cm}^{-3}\right)$. The plasma expansion decreases very strongly the carrier density because it takes place much faster than electron and holes can be created. Thus the lattice heating occurs mainly via carrier diffusion and recombination as analysed above.

3.2 THE RANGE $W<7.5 \mathrm{MW} / \mathrm{cm}^{2}$. - In this range we observe very clearly with OM and SEM the existence of crystallization with minimal power density $W=3.5 \mathrm{MW} / \mathrm{cm}^{2}$. In the figure 3 we plotted the absorbed energy in the sample $E=(1-R) I_{0} \int_{0}^{\infty} F(t) \mathrm{d} t$ against the excitation power density $W$. Both the slope change between the curve $E=f(W)$ and $E_{\mathrm{b}}=f(W)$ and the absence of the dip in the diffracted pulses suggest the existence of the crystallization mechanism 
different from the one in the range $W>7.5 \mathrm{MW} / \mathrm{cm}^{2}$. This mechanism would proceed without melting. Analogous conclusion has already been proposed from microscope observations [28-29]. Besides under relatively weak free carrier concentration $\left(10^{19} \mathrm{~cm}^{-3}\right)$ at room temperature, the predominant mechanisms are the radiative and surface recombination and the latter one has a high recombination rate [30]. The influence of surface recombination on lattice heating can be important, but sufficiently large crystallized grains and measurements of the efficiency of the grating obtained after crystallization (3-4\%) show that a relatively important thickness of about $0.12 \mu$ is crystallized.

A question might be set about the absence of the dip at low excitations. Isn't it due to a lack of sensitivity of our method when the melted zone is very thin ? In fact we are able to detect a phase change of 0.05 . Whereas no data on $n$ for liquid GaAs are available, it seems reasonable to check our method sensitivity in using the value of $n_{1}$ for liquid $\mathrm{Si}$ [31]. If we assume a similar change in $n$ for GaAs, we could detect a melted zone thickness as low as $200 \AA$.

\section{Conclusion.}

In summary, the experimental results indicate that under high excitations the free carrier diffusion is a predominant process in laser heating for $\mathrm{ns}$ scale. In the laser annealing process of a-GaAs, we have observed two behaviours : under high excitation $\left(W>7.5 \mathrm{MW} / \mathrm{cm}^{2}\right)$ the process is related to a liquid phase crystallization (LP-CR) ; under weak rate of excitation $\left(3.5 \mathrm{MW} / \mathrm{cm}^{2}<\right.$ $W<7.5 \mathrm{MW} / \mathrm{cm}^{2}$ ) the crystallization proceeds without melting (SP-CR).

\section{Acknowledgments.}

We are grateful to J. Bok, M. Combescot and C. Benoit à la Guillaume for helpful and stimulating discussions. We would like to thank $H$. Carchano for providing us with the a-GaAs samples and L. Laude and P.' Mathiez for valuable suggestions.

\section{References}

[1] Woerdman, J. P., Philips Res. Rep. Suppl. № 7 (1971).

[2] Hoffman, C. A., Jarasiunas, K., Gerritsen, H. J. and Nurmikxo, A., Appl. Phys. Lett. 33 (1978) 536.

[3] Vaitkus, Yu., Gaubus, E. and Yarachyunas, K., Sov. Phys. Solid State 20 (1978) 1824.

[4] Smirl, A. L., Moss, S. C. and Lindle, J. R., Phys. Rev. B 25 (1982) 2645.

[5] Eichler, H. J. and Massmann, F., J. Appl. Phys. 54 (1982) 3237.

[6] Enns, R. H. and Rangnekar, S. S., Can. J. Phys. 52 (1974) 99.

[7] Apanasevich, P. A. and Afanas'ev, A. A., Sov. Phys. Solid State 18 (1976) 570.

[8] Yu, P. Y. and Cardona, M., Phys. Rev. B 2 (1970) 3193.

[9] KLeIN, W. R. and COOK, B. D., IEEE Trans. Sonics Ultrason. 14 (1967) 123.

[10] Rosenthal, W., Solid State Commun. 13 (1973) 1215.

[11] Benz, G. and Conradt, R., Phys. Rev. B 16 (1977) 843.

[12] Stern, F., Phys. Rev. A 133 (1963) 1653.

[13] TeschKe, O., Rev. Bras. Fis. 10 (1980) 45.

'[14] Косh, S. W., Schmitt-KINK, S. and Haug, H., Solid State Commun. 38 (1981) 1026.

[15] Baeri, P., Campisano, S. U., Foti, G. and Rimini, E., J. Appl. Phys. 50 (1979) 788.

[16] Murakami, K., Tokita, K. and Masuda, K., Japan. J. Appl. Phys. 20 (1981) L 867.

[17] Glasov, V. M., Chizhevskaya, S. N. and Glagoleva, N. N., Liquid Semiconductors (Plenum Press, New York) 1969.

[18] Auston, D. H., Golovchenko, J. A., Simons, A. L. and Surko, C. M., Appl. Phys. Lett. 34 (1979) 777.

[19] Bhattacharyya, A., Streetman, B. G. and Hess, K., J. Appl. Phys. 52 (1980) 3611.

[20] Gallant, M. I. and van Driel, H. M., Phys. Rev. B 26 (1982) 2133.

[21] Young, J. F. and van Driel, H. M., Phys. Rev. B 26 (1982) 2147. 
[22] Neuberger, M., Handbook of Electronic Materials (IFI/Plenum, New York) 1970, Vol. 2.

[23] Аmith, A., Kudman, I. and Steigmeier, E. F., Phys. Rev. A 138 (1965) 1270.

[24] Lichter, B. D. and Sommelet, P., Trans. Metall. Soc. AIME 245 (1969) 1021.

[25] Ref. [22], Vol. 5.

[26] Auston, D. H. and Shank, G. V., Phys. Rev. Lett. 32 (1974) 1120.

[27] Combescot, M., Phys. Lett. A 85 (1981) 308.

[28] Nojima, S., J. Appl. Phys. 53 (1982) 5028.

[29] Bensahel, D. and Auvert, G., Proceedings of the Material Research Society Meeting, Boston, Nov. 1982 (to be published).

[30] Jastrazebski, L., LAGowski, J. and Gatos, Appl. Phys. Lett. 27 (1975) 537.

[31] Shvarev, K. M., Baum, B. A. and Gel'D, P. V., Sov. Phys. Solid State 16 (1975) 2111. 